\title{
Paroxetine versus Venlafaxine and Escitalopram in Korean Patients with Major Depressive Disorder: A Randomized, Rater-blinded, Six-week Study
}

\author{
Young Sup Woo ${ }^{1}$, Roger S. McIntyre ${ }^{2,3}$, Jung-Bum Kim ${ }^{4}$, Min-Soo Lee ${ }^{5}$, Jae-Min Kim ${ }^{6}$, Hyeon Woo Yim ${ }^{7}$, \\ Tae-Youn Jun ${ }^{1}$ \\ ${ }^{1}$ Department of Psychiatry, College of Medicine, The Catholic University of Korea, Seoul, Korea, ${ }^{2}$ Mood Disorders Psychopharmacology Unit, \\ University Health Network, ${ }^{3}$ Department of Psychiatry, University of Toronto, Toronto, Ontario, Canada, ${ }^{4}$ Department of Psychiatry, Keimyung \\ University School of Medicine, Daegu, ${ }^{5}$ Department of Psychiatry, Korea University College of Medicine, Seoul, ${ }^{6}$ Department of Psychiatry, \\ Chonnam National University Medical School, Gwangju, 'Department of Preventive Medicine, College of Medicine, The Catholic University of \\ Korea, Seoul, Korea
}

\begin{abstract}
Objective: The purpose of this study was to compare the efficacy and safety of escitalopram, paroxetine and venlafaxine in Korean patients with major depressive disorder (MDD).

Methods: A total of 449 Korean MDD patients were recruited in a six-week, randomized, rater-blinded, active-controlled trial and were evenly randomized to paroxetine, venlafaxine, or escitalopram treatment.

Results: When comparing the mean difference for the Montgomery-Aisberg Depression Rating Scale (MADRS) and the Hamilton Depression Rating Scale (HDRS) total scores during six weeks, paroxetine $(-6.4 \pm 0.4$, and $-5.4 \pm 0.4$, respectively) was found to be significantly superior to escitalopram $(-3.7 \pm 0.5$ and $-3.1 \pm 0.4$, respectively). Venlafaxine had a significantly lower MADRS total score $(-5.4 \pm 0.4)$ than escitalopram. When adjusting baseline variables, the response, according to the MADRS and HDRS scores, in the paroxetine group was greater than that for the escitalopram group (odds ratio $[\mathrm{OR}]=2.43,95 \%$ confidence interval $[\mathrm{Cl}]=1.42-4.16$ for MADRS; and OR=2.32, 95\% Cl=1.35-3.97 for HDRS) and the venlafaxine group (OR=1.94, 95\% Cl=1.17-3.21 for MADRS; and $\mathrm{OR}=1.71,95 \% \mathrm{Cl}=1.03-2.83$ for HDRS). Despite that the overall tolerability was high and similar among the three groups, a total of 268 subjects (59.7\%) prematurely discontinued treatment, representing the main limitation of the present study. Conclusion: Although a low study completion rate limits generalizability, our findings suggest that paroxetine might be superior to escitalopram in Korean MDD patients. Further studies should be conducted to draw a definite conclusion.
\end{abstract}

KEY WORDS: Paroxetine; Venlafaxine; Escitalopram; Major depressive disorder; Korean.

\section{INTRODUCTION}

Major depressive disorder (MDD) affects every country ubiquitously. However, there are remarkable differences in the prevalence rates of MDD. Previous epidemiological studies in East Asia have consistently reported a lower prevalence of MDD than have Western, and, particularly, United States (US)-based epidemiological studies. ${ }^{1-4)}$ In a cross-national comparison of MDD prevalence, ${ }^{5}$ lifetime prevalence of MDD was found to be $1.5 \%$ in Taiwan and $2.9 \%$ in Korea, substantially lower than the $5.2-16.4 \%$

\footnotetext{
Received: December 22, 2016 / Revised: March 15, 2017

Accepted: March 17, 2017

Address for correspondence: Tae-Youn Jun, MD, PhD Department of Psychiatry, Yeouido St. Mary's Hospital, College of Medicine, The Catholic University of Korea, 10 63-ro, Yeongdeungpo-gu, Seoul 07345, Korea

Tel: +82-2-3779-1250, Fax: +82-2-780-6577

E-mail: tyjun@catholic.ac.kr
}

prevalence found in Western countries such as the US, Canada, France, Italy, New Zealand and Germany. Accordingly, in a recent systematic review, ${ }^{4)}$ estimates of MDD prevalence in East and Southeast Asia were much lower than other regions, even after adjusting for methodological differences.

Not only can differences in prevalence rates of depressive disorders be identified, but the profile and expression of depressive symptoms also vary among ethnic/racial groups. East Asians with depression tend to emphasize somatic complaints and conceptualize their illness as a physical rather than mental ailment, ${ }^{6}$ while displaying a significantly lower level of positive affects compared to non-Eastern subjects. ${ }^{7,8)}$ Moreover, studies have shown that there are cross-ethnic variations in the biological aspects of MDD. Existing studies have shown that Asians tend to metabolize many psychotropic drugs slower compared to Caucasians. Thus, Asian patients tend to show a

(c) This is an Open-Access article distributed under the terms of the Creative Commons Attribution Non-Commercial License (http://creativecommons.org/licenses/by-nc/4.0) which permits unrestricted non-commercial use, distribution, and reproduction in any medium, provided the original work is properly cited 
greater therapeutic response and experience adverse events (AE) at lower dosages than their Caucasian counterparts. ${ }^{9-11)}$ Pharmacogenetic studies have also suggested ethnic/racial differences in the polymorphisms in those genes controlling the function of neurotransmitter systems thought to be related to the pathogenesis and treatment response in MDD. ${ }^{12-16)}$

In the aggregate, the literature has supported the idea that ethnicity/race represents some important factors in determining psychotropic responses. Consequently, a number of countries, including Asian countries, have developed their own treatment guidelines for MDD. ${ }^{17-20)}$ In Asia, however, a shortage of local evidence-based information is the primary problem in developing treatment guidelines. Most of the guidelines are based on a consensus of opinion derived from Western research data and guidelines. ${ }^{21)}$ Only a few randomized, controlled trials of MDD treatment have been conducted in Asian populations, and most studies conducted were sponsored by the pharmaceutical industry.

Herein, we conducted a randomized, rater-blinded study to compare the efficacy and safety of escitalopram, paroxetine and venlafaxine, three of the most commonly prescribed antidepressants in Korea, to provide evidence for the treatment of MDD in Korean patients.

\section{METHODS}

\section{Participants}

Men and women aged 18 to 65 years with a primary diagnosis of MDD without psychotic features, as defined by the Diagnostic and Statistical Manual of Mental Disorders, fourth edition (DSM-IV), were eligible for enrollment. Inclusion criteria included patients who did not receive adequate antidepressant treatment, defined as $\geq 4$ consecutive weeks of treatment at the recommended dosage for the particular antidepressant, and for a current major depressive episode with a minimum 17-item Hamilton Depression Rating Scale (HDRS) with a total score $\geq 14$ at baseline. Those with a current or past comorbid diagnosis of schizophrenia, schizoaffective disorder, schizophreniform disorder, a psychotic disorder not otherwise specified, bipolar disorder, alcohol or substance dependence, dementia, an eating disorder, obsessive compulsive disorder, or other significant medical/neurological conditions, those who had been treated previously with electroconvulsive therapy for the current episode, those who were currently pregnant/breastfeeding or with an active suicidal risk were excluded. Subjects with an unclear his- tory of antidepressant treatment prior to the study entry were also excluded.

\section{Treatment Protocol}

This was a 6-week, prospective, randomized, raterblinded, active-controlled trial conducted from September 2008 through to December 2013 at six university hospitals across the Republic of Korea. Eligible subjects were randomized in a 1:1:1 ratio to one of three treatment arms: paroxetine, venlafaxine, or escitalopram. Drug dosages and titration schedules were based on the recommendations of the prescribing information for each product and according to the judgment of the clinicians involved in the study. No other psychotropic drugs were allowed during the study period, except benzodiazepines (up to 4 $\mathrm{mg} /$ day of lorazepam or equivalent) and hypnotics (up to $10 \mathrm{mg} /$ day of zolpidem or equivalent).

\section{Assessments}

Study patients were assessed at baseline, 1, 2, 3, and 6 weeks. The main outcome measure was the MontgomeryÅsberg Depression Rating Scale (MADRS) change during the 6 weeks of the study. Response was defined as a MADRS/HDRS score improvement greater than $50 \%$ of the baseline score and remission as 12 or less for the MADRS total score, and 7 or less for the HDRS total score. In addition, we used a 3 -factor model for the MADRS proposed by Suzuki et al. ${ }^{22)}$ : factor 1, defined by three items representing dysphoria, i.e., reported sadness, pessimistic thoughts, and suicidal thoughts; factor 2, defined by four items representing retardation, i.e., lassitude, inability to feel, apparent sadness, and concentration difficulties; and factor 3 , defined by three items representing vegetative symptoms, i.e., reduced sleep, reduced appetite, and inner tension. Other instruments used were the Clinical Global Impression-Severity (CGI-S) and Improvement (CGI-I), the Global Assessment of Functioning (GAF), and the Sheehan Disability Scale (SDS). ${ }^{23)}$ All assessors received the same investigators' training module and were blinded to the patients' conditions and prescribed medications.

Safety was assessed via AEs, vital signs, weight, and physical examination findings at each visit. AEs during the study period were recorded by clinical research coordinators using the Udvalg for Kliniske Undersogelser (UKU) side-effect rating scale ${ }^{24)}$ and evaluated for severity and the causal relationship to the study drug. 


\section{Statistical Analysis}

Subjects who were randomized and received one or more doses of study drug and had one or more post-baseline values for the primary efficacy assessment were included in the analysis set. We compared baseline demographic and clinical characteristics data among the paroxetine, venlafaxine and escitalopram groups. We compared continuous variables such as age and scores on depressive symptom scales among the three groups using an analysis of variance (ANOVA) and categorical variables (such as sex and presence or absence of previous episodes) using the chi-square test, or Fisher's exact test when cell sizes were small.

The primary endpoint (least squares mean change in the MADRS total score from baseline) was based on a mixed model for repeated measures analysis of covariance (ANCOVA) with treatment group as the between-subject factor, and age, sex, baseline MADRS score, mean dose of antidepressant during the study (fluoxetine equivalent dose/kg/day), regional center variability, and variables that were significantly different at baseline comparison (first onset vs. recurrent depression) as covariates. Secondary variables (HDRS, CGI-S, GAF, and SDS) were analyzed in a similar manner to the primary endpoint. Response and remission rates were analyzed by multivariate logistic regression, having the same structure as the ANCOVA described above. Missing values were inputted using the last observation carried forward approach.

Serious adverse events (SAEs) were recorded from the date the informed consent was obtained to the last follow-up contact, and other AEs were documented from the beginning of drug administration to the end of the follow-up period. In the present analysis, cases with item severity scores $\geq 2$ and a level of association score of 3 in UKU were considered as experiencing drug-related AEs. AEs leading to discontinuation of the study drug or withdrawal from the study were also documented.

Statistical significance was set at $p<0.05$ (two-tailed) for all tests. All statistical analyses were conducted using the Statistical Analysis System software package (SAS, version 9.1; SAS Institute, Inc., Cary, NC, USA).

\section{Ethics}

The study was conducted according to the Declaration of Helsinki and Good Clinical Practice. Written informed consent was obtained from all subjects after the subjects were given an extensive explanation of the nature and procedures of the study. The study protocol was approved by the institutional review or ethics committees at each study site.

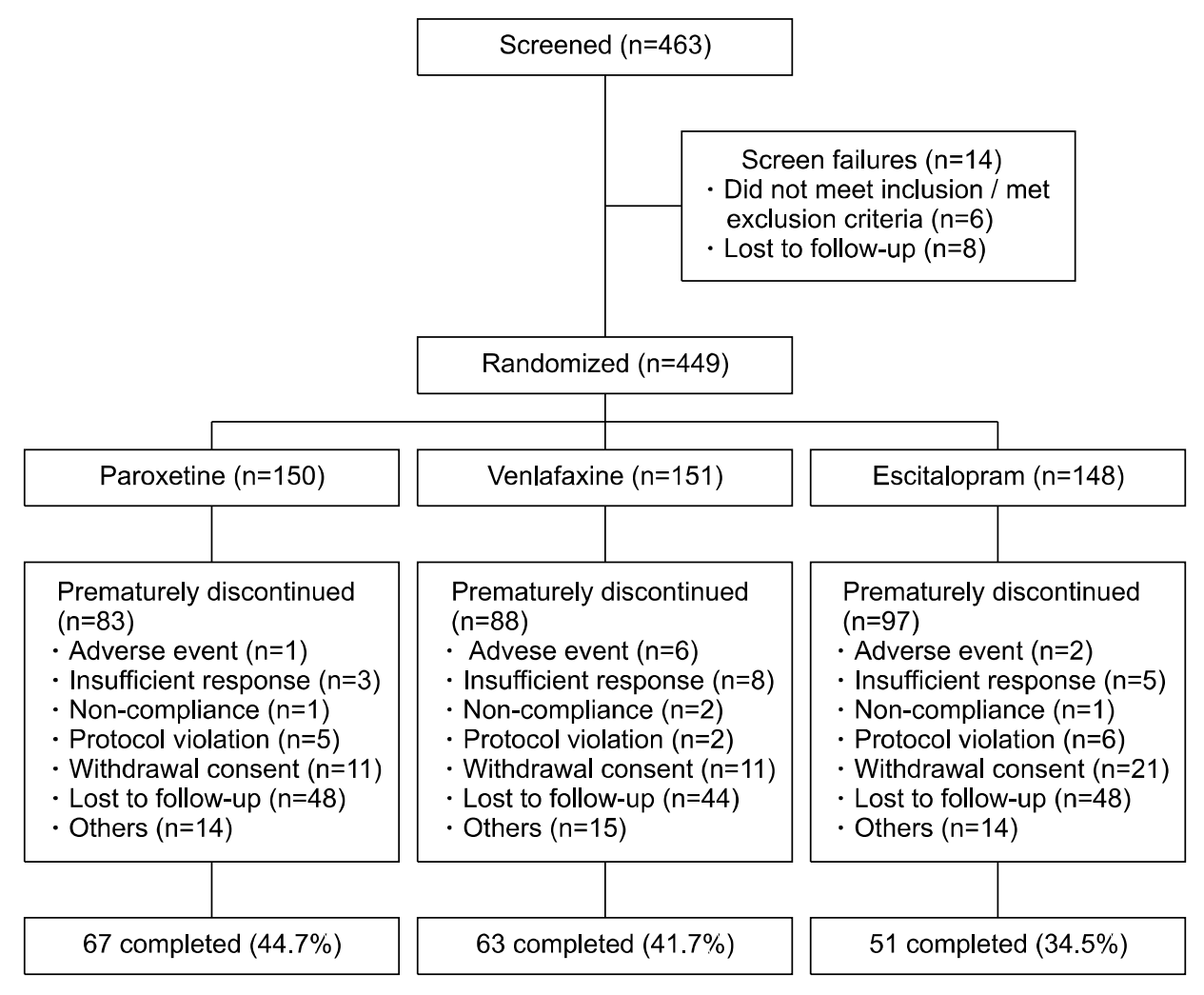

Fig. 1. Subject disposition. 


\section{RESULTS}

\section{Characteristics of the Sample}

Of a total of 463 patients screened for the present study (Fig. 1), 449 met inclusion and exclusion criteria for the analysis, and were randomized and received the study drug (paroxetine, $\mathrm{n}=150$; venlafaxine, $\mathrm{n}=151$; escitalopram, $\mathrm{n}=148$ ). Two hundred and sixty-eight subjects prematurely discontinued treatment, with the most common reasons being lost to follow-up $(n=140)$, withdrawal of consent ( $\mathrm{n}=43)$, insufficient treatment response $(\mathrm{n}=16)$, protocol violation $(n=13)$, or AEs $(n=9)$. The baseline sociodemographic and clinical characteristics of the subjects are summarized in Table 1. Mean MADRS total score of total subjects at baseline was 26.6 and the mean HDRS score was 21.3, indicating that subjects overall experienced moderately severe events. In the CGI-S, the mean score was 4.6 indicating that patients were overall moderately to markedly ill.

When comparing baseline characteristics of the three groups, there was no significant difference in socio-demographic and clinical characteristics among them (Table 1). The proportion of males and patients experiencing the first onset of depression differed among the three groups with a trend-level significance $(p<0.010)$. Men constituted $28.5 \%(n=43)$ of the venlafaxine group, $21.3 \%(n=32)$ of the paroxetine group, and $18.2 \%(\mathrm{n}=27)$ of the escitalopram group $(p=0.095)$, and those experiencing first-onset depression were $70.3 \%(\mathrm{n}=104)$ in the escitalopram group, $63.6 \%(\mathrm{n}=96)$ in the venlafaxine group, and $57.4 \%$ $(\mathrm{n}=86)$ in the paroxetine group $(p=0.067)$. There was no significant difference in total scores of HDRS $(p=0.584)$ and MADRS $(p=0.477)$, CGI-S $(p=0.224)$, GAF $(p=0.546)$, and SDS $(p=0.172)$ scores. The MADRS factor scores (dysphoria factor, $p=0.760$; retardation factor, $p=0.324$, vegetative symptom factor, $p=0.315$ ) and MADRS individual item scores at baseline were not significantly

Table 1. Demographic and clinical characteristics

\begin{tabular}{|c|c|c|c|c|c|c|}
\hline \multicolumn{2}{|c|}{ Characteristic } & \multirow{2}{*}{$\begin{array}{c}\begin{array}{c}\text { Total } \\
(n=449)\end{array} \\
102(22.7)\end{array}$} & \multirow{2}{*}{$\begin{array}{l}\begin{array}{l}\text { Paroxetine } \\
(n=150)\end{array} \\
32(21.3)\end{array}$} & \multirow{2}{*}{$\begin{array}{l}\begin{array}{l}\text { Venlafaxine } \\
(n=151)\end{array} \\
43(28.5)\end{array}$} & \multirow{2}{*}{$\begin{array}{l}\begin{array}{c}\text { Escitalopram } \\
(n=148)\end{array} \\
27(18.2)\end{array}$} & \multirow{2}{*}{$\frac{p \text { value }}{0.095}$} \\
\hline Male & & & & & & \\
\hline \multirow[t]{3}{*}{ Age (yr) } & $<30$ & $83(18.5)$ & $26(17.3)$ & $32(21.2)$ & $25(16.9)$ & \multirow[t]{3}{*}{0.710} \\
\hline & $\geq 30,<50$ & $152(33.9)$ & $56(37.3)$ & $47(31.1)$ & $49(33.1)$ & \\
\hline & $\geq 50$ & $214(47.7)$ & $68(45.3)$ & $72(47.7)$ & $74(50.0)$ & \\
\hline Married & & $295(65.7)$ & $102(68.0)$ & $94(62.3)$ & $99(66.9)$ & 0.537 \\
\hline Employed & & $160(35.6)$ & $47(31.3)$ & $61(40.4)$ & $52(35.1)$ & 0.237 \\
\hline Low family income & DO USD/mo) & $172(38.3)$ & $50(33.3)$ & $65(43.0)$ & $57(38.5)$ & 0.222 \\
\hline \multirow[t]{5}{*}{ Level of education } & Primary school & $91(20.6)$ & $30(20.0)$ & $30(19.9)$ & $31(21.1)$ & \multirow[t]{5}{*}{0.852} \\
\hline & Middle school & $82(18.6)$ & $22(14.7)$ & $28(18.5)$ & $32(21.8)$ & \\
\hline & High school & $155(35.1)$ & $54(36.0)$ & $53(35.1)$ & $48(32.7)$ & \\
\hline & Post-secondary & $114(25.8)$ & $41(27.3)$ & $37(24.5)$ & $36(24.5)$ & \\
\hline & Not available & $7(1.6)$ & $3(2.0)$ & $3(2.0)$ & $1(0.7)$ & \\
\hline \multirow[t]{3}{*}{ Age at onset (yr) } & $<30$ & $49(10.9)$ & $18(12.0)$ & $19(12.6)$ & $12(8.1)$ & \multirow[t]{3}{*}{0.609} \\
\hline & $\geq 30,<50$ & $360(80.2)$ & $118(78.7)$ & $117(77.5)$ & $125(84.5)$ & \\
\hline & $\geq 50$ & $40(8.9)$ & $14(9.3)$ & $15(9.9)$ & $11(7.4)$ & \\
\hline \multirow{4}{*}{\multicolumn{2}{|c|}{$\begin{array}{l}\text { First onset depression } \\
\text { Number of past depressive episodes } \\
\text { Severe depression (baseline MADRS >32) } \\
\text { Family history of depression }\end{array}$}} & $286(63.7)$ & $86(57.3)$ & $96(63.6)$ & $104(70.3)$ & 0.067 \\
\hline & & $1.6 \pm 1.3$ & $1.6 \pm 1.4$ & $1.6 \pm 1.3$ & $1.7 \pm 1.1$ & 0.959 \\
\hline & & $94(20.9)$ & $30(20.0)$ & $25(16.6)$ & $39(26.4)$ & 0.108 \\
\hline & & $68(15.1)$ & $25(16.7)$ & $22(14.6)$ & $21(14.2)$ & 0.828 \\
\hline \multicolumn{2}{|c|}{ Current physical comorbidity at baseline } & $151(33.6)$ & $48(32.0)$ & $50(33.1)$ & $53(35.8)$ & 0.774 \\
\hline \multicolumn{2}{|c|}{ Benzodiazepine use at baseline } & $293(65.3)$ & $99(66.0)$ & $91(60.3)$ & $103(69.6)$ & 0.232 \\
\hline \multicolumn{2}{|c|}{$\begin{array}{l}\text { Dose of antidepressants } \\
\text { (fluoxetine equivalent, mg/day) }\end{array}$} & $22.6 \pm 10.5$ & $19.8 \pm 9.0$ & $20.4 \pm 11.0$ & $27.7 \pm 9.4$ & $<0.001^{*}$ \\
\hline \multicolumn{2}{|c|}{$\begin{array}{l}\text { Dose of antidepressants } \\
\text { (fluoxetine equivalent, mg/kg/day) }\end{array}$} & $0.4 \pm 0.2$ & $0.3 \pm 0.2$ & $0.3 \pm 0.2$ & $0.5 \pm 0.2$ & $<0.001^{*}$ \\
\hline \multirow[t]{5}{*}{ Baseline scores } & HDRS & $21.3 \pm 4.7$ & $21.0 \pm 4.8$ & $21.6 \pm 4.5$ & $21.3 \pm 4.9$ & 0.584 \\
\hline & MADRS & $26.6 \pm 7.1$ & $26.0 \pm 6.6$ & $26.9 \pm 6.6$ & $26.9 \pm 8.2$ & 0.477 \\
\hline & CGI-S & $4.6 \pm 1.0$ & $4.5 \pm 0.9$ & $4.6 \pm 1.0$ & $4.6 \pm 1.0$ & 0.224 \\
\hline & GAF & $59.1 \pm 7.9$ & $58.9 \pm 7.2$ & $59.7 \pm 8.1$ & $58.8 \pm 8.4$ & 0.546 \\
\hline & SDS & $17.8 \pm 6.5$ & $18.4 \pm 6.6$ & $17.9 \pm 6.0$ & $17.0 \pm 6.8$ & 0.172 \\
\hline
\end{tabular}

Values are presented as number (\%), mean \pm standard deviation, or median (range).

USD, US dollar; HDRS, 17-item Hamilton Depression Rating Scale; MADRS, Montgomery-Åsberg Depression Rating Scale; CGI-S, Clinical Global Impression- Severity; GAF, Global Assessment of Functioning; SDS, Sheehan Disability Scale.

*Significant differences between the three groups $(p<0.05)$. 
Table 2. Baseline MADRS factor and item score

\begin{tabular}{|c|c|c|c|c|c|c|c|}
\hline \multirow{2}{*}{ Factor } & \multirow{2}{*}{$\begin{array}{l}\text { Paroxetine } \\
\qquad(n=150)\end{array}$} & \multirow{2}{*}{$\begin{array}{l}\text { Venlafaxine } \\
\qquad(n=151)\end{array}$} & \multirow{2}{*}{$\begin{array}{l}\text { Escitalopram } \\
\qquad(n=148)\end{array}$} & \multicolumn{4}{|c|}{$p$ value } \\
\hline & & & & 1 vs. 2 vs. 3 & 1 vs. 2 & 1 vs. 3 & 2 vs. 3 \\
\hline Dysphoria factor & $7.9 \pm 2.7$ & $7.7 \pm 3.1$ & $7.8 \pm 2.8$ & 0.760 & $>0.999$ & $>0.999$ & $>0.999$ \\
\hline 2. Reported sadness & $3.5 \pm 1.1$ & $3.4 \pm 1.3$ & $3.3 \pm 1.2$ & 0.327 & 0.468 & $>0.999$ & 0.792 \\
\hline 9. Pessimistic thoughts & $2.3 \pm 1.0$ & $2.3 \pm 1.2$ & $2.4 \pm 1.2$ & 0.537 & $>0.999$ & $>0.999$ & 0.795 \\
\hline 10. Suicidal thoughts & $2.1 \pm 1.3$ & $2.0 \pm 1.4$ & $2.1 \pm 1.4$ & 0.562 & $>0.999$ & $>0.999$ & 0.928 \\
\hline Retardation factor & $10.6 \pm 3.2$ & $10.8 \pm 3.7$ & $10.2 \pm 3.0$ & 0.324 & 0.943 & $>0.999$ & 0.428 \\
\hline 1. Apparent sadness & $3.1 \pm 1.0$ & $3.1 \pm 1.0$ & $2.9 \pm 1.1$ & 0.387 & 0.560 & $>0.999$ & 0.961 \\
\hline 6. Concentration difficulties & $2.3 \pm 1.1$ & $2.4 \pm 1.2$ & $2.3 \pm 1.0$ & 0.632 & $>0.999$ & $>0.999$ & 1.000 \\
\hline 7. Lassitude & $2.7 \pm 1.1$ & $2.7 \pm 1.2$ & $2.5 \pm 1.1$ & 0.383 & $>0.999$ & $>0.999$ & 0.538 \\
\hline 8. Inability to feel & $2.5 \pm 1.3$ & $2.6 \pm 1.4$ & $2.4 \pm 1.2$ & 0.364 & 0.849 & $>0.999$ & 0.540 \\
\hline Vegetative symptom factor & $8.4 \pm 2.8$ & $8.5 \pm 3.0$ & $8.0 \pm 2.8$ & 0.315 & 0.758 & $>0.999$ & 0.452 \\
\hline 3. Inner tension & $2.8 \pm 0.9$ & $2.9 \pm 1.1$ & $2.8 \pm 0.9$ & 0.816 & $>0.999$ & $>0.999$ & $>0.999$ \\
\hline 4. Reduced sleep & $3.2 \pm 1.6$ & $3.4 \pm 1.6$ & $3.0 \pm 1.6$ & 0.088 & $>0.999$ & 0.400 & 0.095 \\
\hline 5. Reduced appetite & $2.4 \pm 1.5$ & $2.1 \pm 1.6$ & $2.1 \pm 1.4$ & 0.291 & 0.493 & 0.552 & $>0.999$ \\
\hline
\end{tabular}

Values are presented mean \pm standard deviation.

MADRS, Montgomery-Åsberg Depression Rating Scale.

Table 3. Primary and secondary endpoints

\begin{tabular}{|c|c|c|c|c|c|c|c|}
\hline \multirow{2}{*}{ Variable } & \multirow{2}{*}{$\begin{array}{l}\text { Paroxetine } \\
(n=150)\end{array}$} & \multirow{2}{*}{$\begin{array}{l}\text { Venlafaxine } \\
\qquad(n=151)\end{array}$} & \multirow{2}{*}{$\begin{array}{l}\text { Escitalopra } \\
m(n=148)\end{array}$} & \multicolumn{3}{|c|}{ Difference ( $p$ value) } & \multirow{2}{*}{$\frac{p \text { value }}{1 \text { vs. } 2 \text { vs. } 3}$} \\
\hline & & & & 1 vs. 2 & 1 vs. 3 & 2 vs. 3 & \\
\hline \multicolumn{8}{|c|}{ Change at week 6, LS } \\
\hline MADRS & $-6.3 \pm 0.4$ & $-5.3 \pm 0.4$ & $-3.8 \pm 0.5$ & $-1.0 \pm 0.6(0.098)$ & $-2.5 \pm 0.6\left(<0.001^{* *}\right)$ & $-1.5 \pm 0.6(0.017)$ & $0.001^{* *}$ \\
\hline HDRS & $-5.3 \pm 0.4$ & $-4.3 \pm 0.4$ & $-3.3 \pm 0.4$ & $-1.0 \pm 0.5(0.045)$ & $-2.1 \pm 0.5\left(<0.001^{* *}\right)$ & $-1.1 \pm 0.5(0.034)$ & $<0.001^{\star *}$ \\
\hline CGI-S & $-0.8 \pm 0.1$ & $-0.7 \pm 0.1$ & $-0.5 \pm 0.1$ & $-0.2 \pm 0.1 \quad(0.086)$ & $-0.3 \pm 0.1\left(0.001^{* *}\right)$ & $-0.2 \pm 0.1 \quad(0.098)$ & $0.005^{\star *}$ \\
\hline \multicolumn{8}{|l|}{ Response } \\
\hline MADRS & $58(38.7)$ & $39(25.8)$ & $40(27.0)$ & $12.9(0.023)$ & $11.7(0.024)$ & $-1.2(0.978)$ & $0.029^{*}$ \\
\hline HDRS & $56(37.3)$ & $41(27.2)$ & $40(27.0)$ & $10.1(0.076)$ & $10.3(0.044)$ & $0.2(0.814)$ & 0.082 \\
\hline \multicolumn{8}{|l|}{ Remission } \\
\hline MADRS & $51(34.0)$ & $43(28.5)$ & $39(26.4)$ & $5.5(0.358)$ & $7.6(0.122)$ & $2.1(0.532)$ & 0.295 \\
\hline HDRS & $33(22.0)$ & $22(14.6)$ & $21(14.2)$ & $7.4(0.112)$ & $7.8(0.067)$ & $0.4(0.813)$ & 0.124 \\
\hline
\end{tabular}

Values are presented as mean \pm standard error or number (\%).

MADRS, Montgomery-Åsberg Depression Rating Scale; HDRS, 17-item Hamilton Depression Rating Scale; CGI-S, Clinical Global ImpressionSeverity.

Least square (LS) mean change was adjusted for age, sex, baseline MADRS score, mean fluoxetine equivalent dose, site, HDRS item 15, and recurrence.

*Significant differences among the three groups $(p<0.05)$; ${ }^{* *}$ significant differences between the two groups (Bonferroni correction, $\left.p<0.017\right)$.

different among the three groups (Table 2).

When comparing mean fluoxetine equivalent $\operatorname{dos}^{25)}$ per weight of each group during the study period, a significant difference was found. The mean fluoxetine equivalent dose was $0.3 \pm 0.2 \mathrm{mg} / \mathrm{kg} /$ day in the paroxetine group, $0.3 \pm 0.2 \mathrm{mg} / \mathrm{kg} / \mathrm{day}$ in the venlafaxine group, and $0.5 \pm 0.2$ $\mathrm{mg} / \mathrm{kg} /$ day in the escitalopram group $(p<0.001)$. The mean lorazepam equivalent dose during the study period was not significantly different among the groups; $1.1 \pm 0.6$ $\mathrm{mg}$ /day in the paroxetine group, $1.2 \pm 1.0 \mathrm{mg} /$ day in the escitalopram group, and $1.1 \pm 0.7 \mathrm{mg} /$ day in venlafaxine group ( $p=0.802)$.

\section{Efficacy}

In the primary efficacy analysis, paroxetine and ven- lafaxine were found to be significantly superior to escitalopram (Table 3). The mean difference was -2.7 (standard error $[\mathrm{SE}] \pm 0.6 ; p<0.001$ ) between paroxetine and escitalopram, and $-1.7 \pm 0.6(p=0.010)$ between venlafaxine and escitalopram for the MADRS total score at week 6 . The difference between paroxetine and venlafaxine in MADRS change from baseline to week $6(-1.0 \pm$ $0.6 ; p=0.106$ ) was not significant. For the HDRS total score, the difference between paroxetine and escitalopram $(-2.3 \pm 0.5 ; p<0.001)$ was significant, but the difference between paroxetine and venlafaxine $(-1.1 \pm 0.5 ; p=$ $0.036)$, and venlafaxine and escitalopram $(-1.2 \pm 0.5$; $p=0.022$ ) did not reach significance at week 6 after the Bonferroni correction for multiple comparisons $(p<$ 0.017). 
Table 4. Adjusted odds ratio (OR) and 95\% confidence interval (Cl) of treatment outcomes

\begin{tabular}{|c|c|c|c|c|c|c|c|}
\hline \multirow{2}{*}{ Variable } & \multicolumn{3}{|c|}{ Paroxetine $(n=150)$} & \multicolumn{3}{|c|}{ Venlafaxine $(n=151)$} & \multirow{2}{*}{$\frac{\text { Escitalopram }(\mathrm{n}=148)}{\text { OR }(95 \% \mathrm{Cl})}$} \\
\hline & & OR $(95 \% \mathrm{Cl})$ & $p$ value & & OR $(95 \% \mathrm{Cl})$ & $p$ value & \\
\hline \multirow[t]{2}{*}{ MADRS response } & vs. escitalopram & $2.43(1.42-4.16)$ & $0.001^{*}$ & vs. escitalopram & $1.26(0.73-2.19)$ & 0.413 & 1 \\
\hline & vs. venlafaxine & $1.94(1.17-3.21)$ & $0.010^{*}$ & vs. paroxetine & 1 & & \\
\hline \multirow[t]{2}{*}{ HDRS response } & vs. escitalopram & $2.32(1.35-3.97)$ & $0.002^{*}$ & vs. escitalopram & $1.36(0.78-2.35)$ & 0.275 & 1 \\
\hline & vs. venlafaxine & $1.71(1.03-2.83)$ & $0.038^{*}$ & vs. paroxetine & 1 & & \\
\hline \multirow{2}{*}{$\begin{array}{l}\text { MADRS remission } \\
(\leq 12)\end{array}$} & vs. escitalopram & $1.96(1.12-3.45)$ & $0.019^{*}$ & vs. escitalopram & $1.34(0.76-2.36)$ & 0.309 & 1 \\
\hline & vs. venlafaxine & $1.45(0.87-2.46)$ & 0.149 & vs. paroxetine & 1 & & \\
\hline \multirow{2}{*}{$\begin{array}{l}\text { HDRS remission } \\
(\leq 7)\end{array}$} & vs. escitalopram & $2.40(1.24-4.63)$ & $0.009^{*}$ & vs. escitalopram & $1.23(0.61-2.45)$ & 0.562 & 1 \\
\hline & vs. venlafaxine & $1.95(1.05-3.63)$ & $0.034^{*}$ & vs. paroxetine & 1 & & \\
\hline
\end{tabular}

MADRS, Montgomery-Åsberg Depression Rating Scale; HDRS, 17-item Hamilton Depression Rating Scale. Adjusted for age, sex, baseline MADRS score, mean fluoxetine equivalent dose, site, and recurrence. ${ }^{*} p<0.05$.

Table 5. Adjusted least squares mean changes in MADRS factor and item score during 6 weeks

\begin{tabular}{|c|c|c|c|c|c|c|c|}
\hline & \multirow{2}{*}{ Paroxetine } & \multirow{2}{*}{ Venlafaxine } & \multirow{2}{*}{ Escitalopram } & \multicolumn{4}{|c|}{$p$ value } \\
\hline & & & & 1 vs. 2 vs. 3 & 1 vs. 2 & 1 vs. 3 & 2 vs. 3 \\
\hline Dysphoria factor & $-2.1 \pm 0.2$ & $-1.9 \pm 0.2$ & $-1.2 \pm 0.2$ & $<0.001^{\star}$ & 0.247 & $<0.001^{\star}$ & $0.002^{*}$ \\
\hline 2. Reported sadness & $-0.8 \pm 0.1$ & $-0.7 \pm 0.1$ & $-0.5 \pm 0.1$ & $0.002^{*}$ & 0.294 & $0.001^{*}$ & 0.025 \\
\hline 9. Pessimistic thoughts & $-0.6 \pm 0.1$ & $-0.6 \pm 0.1$ & $-0.4 \pm 0.1$ & $0.01^{*}$ & 0.655 & $0.011^{*}$ & 0.034 \\
\hline 10. Suicidal thoughts & $-0.7 \pm 0.1$ & $-0.6 \pm 0.1$ & $-0.3 \pm 0.1$ & $<0.001^{*}$ & 0.334 & $<0.001^{*}$ & $0.003^{*}$ \\
\hline Retardation factor & $-2.1 \pm 0.2$ & $-1.8 \pm 0.2$ & $-1.2 \pm 0.2$ & $0.001^{*}$ & 0.224 & $0.002^{*}$ & 0.043 \\
\hline 1. Apparent sadness & $-0.7 \pm 0.1$ & $-0.6 \pm 0.1$ & $-0.4 \pm 0.1$ & $<0.001^{*}$ & 0.413 & $0.001^{*}$ & $0.007^{*}$ \\
\hline 6. Concentration difficulties & $-0.4 \pm 0.1$ & $-0.4 \pm 0.1$ & $-0.2 \pm 0.1$ & $0.031^{*}$ & 0.462 & 0.028 & 0.129 \\
\hline 7. Lassitude & $-0.5 \pm 0.1$ & $-0.4 \pm 0.1$ & $-0.2 \pm 0.1$ & 0.585 & 0.616 & 0.401 & 0.712 \\
\hline 8. Inability to feel & $-0.5 \pm 0.1$ & $-0.4 \pm 0.1$ & $-0.3 \pm 0.1$ & $0.015^{\star}$ & 0.160 & $0.011^{*}$ & 0.211 \\
\hline Vegetative symptom factor & $-2.1 \pm 0.2$ & $-1.7 \pm 0.2$ & $-1.2 \pm 0.2$ & $<0.001^{*}$ & 0.040 & $<0.001^{*}$ & 0.042 \\
\hline 3. Inner tension & $-0.6 \pm 0.1$ & $-0.5 \pm 0.1$ & $-0.4 \pm 0.1$ & $0.027^{*}$ & 0.671 & 0.061 & 0.138 \\
\hline 4. Reduced sleep & $-1.1 \pm 0.1$ & $-0.8 \pm 0.1$ & $-0.6 \pm 0.1$ & $<0.001^{\star}$ & 0.017 & $<0.001^{*}$ & 0.141 \\
\hline 5. Reduced appetite & $-0.5 \pm 0.1$ & $-0.4 \pm 0.1$ & $-0.2 \pm 0.1$ & $0.013^{*}$ & 0.221 & $0.012^{*}$ & 0.167 \\
\hline
\end{tabular}

Values are presented as mean \pm standard error.

MADRS, Montgomery-Åsberg Depression Rating Scale

Adjusted for age, sex, baseline MADRS score, mean fluoxetine equivalent dose, site, and recurrence.

*Significant difference between the three groups $(p<0.05)$; **significant difference between the two groups (significance was adjusted by Bonferroni correction, $p<0.017)$.

The unadjusted MADRS response rate was significantly different among the three treatment groups (Table 3); MADRS response at week 6 was achieved in $38.7 \%(n=58), 25.8 \%(n=39)$, and $27.0 \%(n=40)$ of subjects in the paroxetine, venlafaxine, and escitalopram groups, respectively $\left(\chi^{2}=7.065\right.$, degree of freedom $[d f]=2$, $p=0.029)$. There was no significant difference among the three groups in unadjusted HDRS response rate $\left(\chi^{2}=\right.$ 4.995, $d f=2, p=0.082)$ and MADRS remission $\left(\chi^{2}=2.445\right.$, $d f=2, p=0.295)$ and HDRS remission $\left(\chi^{2}=4.172, d f=2\right.$, $p=0.124$ ) rates (Table 3 ). After adjusting for potential confounding variables, the paroxetine group showed a significantly higher response rate for MADRS (odds ratio $[\mathrm{OR}]=2.43,95 \%$ confidence interval $[\mathrm{CI}]=1.42-4.16, p=$ 0.001) and HDRS (OR=2.32, 95\% CI=1.35-3.97, $p=$ 0.002 ) than the escitalopram group (Table 4). Even when compared to the venlafaxine group, the paroxetine group showed a significantly higher response rate for MADRS $(\mathrm{OR}=1.94,95 \% \mathrm{CI}=1.17-3.21, p=0.010)$ and HDRS $(\mathrm{OR}=1.71,95 \% \mathrm{CI}=1.03-2.83, p=0.038)$. The remission rate was also significantly higher in the paroxetine group than the escitalopram $(\mathrm{OR}=2.40,95 \% \mathrm{CI}=1.24-4.63$, $p=0.009)$ and venlafaxine ( $\mathrm{OR}=1.95,95 \% \mathrm{CI}=1.05-3.63$, $p=0.034$ ) groups for HDRS. The MADRS remission rate was significantly higher in the paroxetine group when compared with the escitalopram group $(\mathrm{OR}=1.96,95 \%$ $\mathrm{CI}=1.12-3.45, p=0.019)$, but was not significantly different between the paroxetine group and the venlafaxine group $(\mathrm{OR}=1.45,95 \% \mathrm{CI}=0.149, p=0.149$; Table 4).

The changes in MADRS factor scores (Table 5) were similar to the changes in the MADRS total score. Paroxetine had greatly reduced scores for all three factors compared to escitalopram with significance (dysphoria factor; $p<0.001$, retardation factor; $p=0.002$, vegetative 
Table 6. Adverse events experienced by $\geq 5 \%$ of subjects

\begin{tabular}{|c|c|c|c|c|}
\hline Adverse event & Full sample $(n=449)$ & Paroxetine $(n=150)$ & Venlafaxine $(n=151)$ & Escitalopram $(n=148)$ \\
\hline Any adverse event & $204(45.4)$ & $72(48.0)$ & $65(43.0)$ & $67(45.3)$ \\
\hline Cognitive impairment & $103(22.9)$ & $37(24.7)$ & $29(19.2)$ & $37(25.0)$ \\
\hline Dysphoric mood & $102(22.7)$ & $34(22.7)$ & $30(19.9)$ & $38(25.7)$ \\
\hline Fatigue & $98(21.8)$ & $34(22.7)$ & $28(18.5)$ & $36(24.3)$ \\
\hline Anxiety & $89(19.8)$ & $32(21.3)$ & $23(15.2)$ & $34(23.0)$ \\
\hline Insomnia & $88(19.6)$ & $32(21.3)$ & $27(17.9)$ & $29(19.6)$ \\
\hline Dry mouth & $83(18.5)$ & $28(18.7)$ & $30(19.9)$ & $25(16.9)$ \\
\hline Sedation & $76(16.9)$ & $26(17.3)$ & $21(13.9)$ & $29(19.6)$ \\
\hline Headache & $63(14.0)$ & $22(14.7)$ & $22(14.6)$ & $19(12.8)$ \\
\hline Constipation & $51(11.4)$ & $19(12.7)$ & $19(12.6)$ & $13(8.8)$ \\
\hline Sexual dysfunction & $34(7.6)$ & $10(6.7)$ & $12(7.9)$ & $12(8.1)$ \\
\hline Palpitation or tachycardia & $31(6.9)$ & $9(6.0)$ & $12(7.9)$ & $10(6.8)$ \\
\hline Increased dream activity & $31(6.9)$ & $8(5.3)$ & $9(6.0)$ & $14(9.5)$ \\
\hline Weight loss/decreased appetite & $30(6.7)$ & $11(7.3)$ & $10(6.6)$ & $9(6.1)$ \\
\hline Increased sweating & $26(5.8)$ & $9(6.0)$ & $7(4.6)$ & $10(6.8)$ \\
\hline Dizziness/orthostatic dizziness & $25(5.6)$ & $6(4.0)$ & $9(6.0)$ & $10(6.8)$ \\
\hline Nausea/vomiting & $22(4.9)$ & $7(4.7)$ & $5(3.3)$ & $10(6.8)$ \\
\hline Weight gain/increased appetite & $21(4.7)$ & $7(4.7)$ & $6(4.0)$ & $8(5.4)$ \\
\hline
\end{tabular}

Values are presented as number (\%).

symptom factor; $p<0.001)$, and venlafaxine had a lower dysphoria factor score than escitalopram $(p=0.002)$.

The changes in GAF score during the 6 weeks in the paroxetine group and venlafaxine group were greater than in the escitalopram group, but the differences were not statistically significant. The mean difference was $2.1 \pm 1.0$ $(p=0.029)$ for paroxetine vs. escitalopram, and $1.9 \pm 1.0$ $(p=0.042)$ for venlafaxine vs. escitalopram. The SDS score decreased from $18.6 \pm 6.4$ at baseline to $15.6 \pm 7.6$ at week 6 in the paroxetine group, from $18.0 \pm 6.1$ to $15.6 \pm 7.1$ in the venlafaxine group, and from $17.1 \pm 6.8$ to $15.5 \pm 7.2$ in the escitalopram group, and was therefore not significantly different among the three groups $(p=0.776)$.

\section{Safety}

The dropout rate was $55.3 \%(\mathrm{n}=83)$ in the paroxetine group, $58.3 \%(\mathrm{n}=88)$ in the venlafaxine group, and $65.5 \%$ $(n=97)$ in the escitalopram group, and was therefore not significantly different among the three treatment groups $\left(\chi^{2}=3.414, d f=2, p=0.181\right)$. A total of 204 patients $(45.4 \%)$ reported 1093 cases of AEs. The percentage of subjects who reported at least one AE during the study period was $48.0 \%, 43.0 \%$, and $45.3 \%$ in the paroxetine, venlafaxine, and escitalopram groups, respectively $\left(\chi^{2}=0.747, d f=2\right.$, $p=0.688$ ). The most frequently reported AEs-which were reported in at least $5 \%$ of the subjects in any of the treatment groups - were cognitive impairment, dysphoric mood, fatigue, anxiety, insomnia, dry mouth, sedation, headache, constipation, sexual dysfunction, palpitation/ tachycardia, increased dream activity, weight loss/de- creased appetite, increased sweating, dizziness/orthostatic dizziness, nausea/vomiting, and weight gain/increased appetite (Table 6). In all treatment groups, the majority of AEs reported were considered to be of mild or moderate severity; 728 cases $(66.6 \%)$ were mild and 321 cases (29.4\%) were moderate. Only 44 cases $(4.0 \%)$ were rated as severe. There were 4 subjects who reported a SAE; 2 cases of hospitalization due to aggravation of depressive symptoms (both of them treated with venlafaxine), and 1 case of suicide attempt in the escitalopram group.

Nine subjects had AEs leading to study discontinuation: $1(0.7 \%), 6(4.0 \%)$, and $2(1.4 \%)$ subjects in the paroxetine, venlafaxine, and escitalopram group, respectively. AEs leading to treatment discontinuation were sexual dysfunction $(\mathrm{n}=1)$ in the paroxetine group, headache $(\mathrm{n}=2)$, fatigue $(\mathrm{n}=2)$, dizziness $(\mathrm{n}=1)$, and blurred vision $(n=1)$ in the venlafaxine group, and insomnia $(n=1)$, and suicide attempt $(n=1)$ in the escitalopram group.

\section{DISCUSSION}

In the present study, paroxetine was found to be superior to escitalopram for the treatment of MDD patients. For several secondary efficacy measures, paroxetine was more effective than venlafaxine in the treatment of MDD. Paroxetine was shown to be superior to venlafaxine and escitalopram according to the response rates measured using MADRS and HDRS scores, and the remission rate measured using the HDRS score when adjusted for baseline variables. This significant superiority of paroxetine 
over escitalopram was also observed in changes from baseline to week 6 according to the adjusted MADRS, HDRS total scores as well as the CGI-S score. Venlafaxine was also superior to escitalopram in the adjusted MADRS total score change.

This result is not coincident with results from previous studies which reported an inferior or comparable antidepressant efficacy of paroxetine compared to escitalopram or venlafaxine. Cipriani et $a l .{ }^{26)}$ reported that escitalopram and venlafaxine were more efficacious than paroxetine. In their meta-analysis which included 117 randomized, controlled trials, the OR for response was 1.35 for escitalopram and 1.27 for venlafaxine. In a recent Cochrane meta-analysis which included a total of 115 randomized, controlled trials, there was no significant difference in antidepressant efficacy when comparing paroxetine to escitalopram or venlafaxine. ${ }^{27)}$

The discrepancy between the results from the present study and previous studies could be attributed to a few contributing factors. First of all, inter-ethnic differences in pharmacogenetics could affect antidepressant responsiveness. ${ }^{28)}$ Variations in gene allele frequencies can contribute to differences in antidepressant responses in different ethnic groups. ${ }^{29)}$ Over $90 \%$ of all drug metabolism could be accounted for by cytochrome P450 (CYP) enzymes, and 1A2, 2D6, 2C9, 2C19, and 3A4 account for $60 \%$ of metabolism. ${ }^{30}$ Escitalopram is metabolized by CYP3A4, CYP2C19 and, to a lesser extent, by CYP2D6, ${ }^{31,32)}$ and paroxetine is mainly metabolized by CYP2D6, while CYP3A4 also contributes to the metabolism of paroxetine. ${ }^{33)}$ As for venlafaxine, CYP2D6 is the major metabolizing enzyme, and its metabolism is partly mediated by CYP3A4 and CYP2C19. ${ }^{34)}$

A marked inter-ethnic difference in the allelic frequencies of CYP genes has been reported, and variants in these genes have been hypothesized to predict variations in antidepressant metabolism, therapeutic responses, and risk of adverse effects. ${ }^{35,36)}$ Most differences between East Asians and Caucasians have been particularly shown in the enzymatic activity of CYP2D6 and the CYP2C subfamily. ${ }^{15)}$ Kirchheiner et al. ${ }^{37)}$ reported that genetic polymorphisms in CYP2D6 or CYP2C19 would require at least a doubling of the dose of extensive metabolizers (EMs) in comparison to poor metabolizers (PMs). Meanwhile, the CYP3A4 exhibits few genetic polymorphisms.

CYP2D6 polymorphism is the most extensively studied oxidation polymorphism in humans. In general, CYP2D6 PMs (who are lacking functional enzymes due to de- fective or deleted genes) reach higher peak serum concentrations and have lower clearances and longer half-lives as compared with CYP2D6 EMs (who are carrying two functional genes). Hence, PMs may have greater susceptibility to adverse effects ${ }^{38)}$ and exhibit lower treatment responses than EMs. ${ }^{39-41)}$ Moreover, the frequency of the phenotype of CYP2D6 PMs differs among ethnic groups. Less than $1 \%$ of Asians, $5 \%$ to $10 \%$ of Caucasians are PMs of CYP2D6. ${ }^{42)}$ Because CYP2D6 is the major enzyme involved in the metabolism of paroxetine and venlafaxine, but is not so extensively involved in the metabolism of escitalopram, the antidepressant efficacy of paroxetine and venlafaxine could be underestimated in studies which include predominantly Caucasians, who have a higher risk of being a PM of paroxetine and venlafaxine than Asians.

Moreover, in a recent cross-ethnic study that investigated the association between serotonin transporter promoter polymorphism (5-HTTLPR) and escitalopram efficacy in depression, ${ }^{43)}$ the response rate for escitalopram treatment was $64 \%$ in Caucasian subjects and $47 \%$ in Koreans. Among those with the 1/1, but not 1/s or s/s genotypes, Caucasian subjects showed higher response and remission rates compared with Koreans. They reported the frequency of the favorable 1 allele in Caucasian as 51\% and $24 \%$ in Koreans, and suggested that it may be expected that Koreans would be less likely to respond to escitalopram due to a lower proportion of 1 alleles in the population, although there have been inconsistent results. ${ }^{44-47)}$

Ethnic differences in depressive symptomatology might also contribute to the discrepancy between results from the present study and other studies, which are mainly from Western countries. It has been known that Asians report more somatic and less affective symptoms of depression, ${ }^{48-51)}$ and high levels of somatic symptoms respond less well to antidepressant treatment. ${ }^{52-54)}$ The association between genetic polymorphisms and somatic symptoms was suggested by Klengel et $_{\text {al }}{ }^{55)}$ who reported an association between serotonin 2A receptor gene (HTR2A) polymorphism (G/G genotype in rs 9534505$)$ and somatization in MDD patients. Moreover, a recent meta-analysis ${ }^{56}$ confirmed that patients with s/s genotype of 5-HTTLPR experience a slower improvement of somatic anxiety symptoms. The frequency of both of $\mathrm{G} / \mathrm{G}$ genotype in HTR2A polymorphism and s/s genotype in 5-HTTLPR is higher in Asian populations than in Caucasians. ${ }^{43,57,58)}$ Asians may show a different or poorer response to antidepressant treatment when compared to Caucasians.

Another issue that should be considered is the dose of antidepressants. Dose equivalence is critically important 
for comparative clinical trials and their meta-analyses, and setting comparable dosages is necessary to facilitate a proper interpretation of results. ${ }^{59)}$ However, in previous studies which reported superiority of escitalopram or a lack of difference in efficacy between antidepressants, categorical dosing classification was used to compare the doses of different antidepressants. ${ }^{26,27)}$ In the present study, we used results from the most recent study to calculate dose equivalents of antidepressants, ${ }^{25)}$ and this might contribute to the inconsistency of the results.

There are a number of limitations that should be considered when interpreting the results from the present study. The most important limitation was the low study completion rate. Only $40 \%$ of subjects completed this sixweek study. The dropout rates for previous studies which compared paroxetine with venlafaxine or escitalopram ranged from $10 \%$ to $26 \%,{ }^{26)}$ indicating this study had an approximately $14 \%$ to $30 \%$ higher dropout rate. The most common reason for dropout in this study was lost to follow-up $(31.2 \%, n=140)$. The dropout rate due to insufficient efficacy $(3.6 \%, n=16)$ or AEs $(2.0 \%, n=9)$ was very low, and this might suggest that the difference in efficacy and safety reported in this study was not a result of the high dropout rate. According to the results from $\mathrm{STAR}^{*} \mathrm{D}$, attrition during antidepressant treatment is related to a number of patient characteristics, including non-white race, lower income, less education and negative attitudes about psychiatric medication. ${ }^{60,61)}$ In this study, the high proportion of low income status (38.3\%), and low educational level (39.2\% below high school) patients might have lowered the study completion rate. Moreover, it is noteworthy that Korean patients have a more negative attitude towards depression than their counterparts in Western countries, ${ }^{62)}$ and this could decrease the completion rate.

The lack of a placebo arm was another limitation in this study. Hence, it was unable to detect the placebo response rate and to exclude the possibility of an improvement in depressive symptoms as part of the natural course of the disease. The absence of assessing inter-rater reliability was another limitation of the present study. In addition, a structured diagnostic interview was not carried out, which might allow for a more detailed diagnosis of psychiatric comorbidities and subtypes of depression. Finally, although the study sample could not be homogenous in terms of type of depression and bipolarity, the issues of bipolarity was not addressed.

The results of this randomized, rater-blinded, six-week, prospective, head-to-head comparison study suggested that paroxetine was more efficacious than escitalopram in overall treatment effects in Korean MDD patients. Paroxetine was found to be superior to venlafaxine in terms of the change in MADRS during six weeks, the response rates for MADRS and HDRS, and the remission rate for HDRS. Venlafaxine had a lower MADRS total score than escitalopram, but a difference could not be identified for the other efficacy measures. Overall tolerability was similar among the three groups. Further studies with a larger cohort are needed to draw definite conclusions on the differences in efficacy among different antidepressants in Asian MDD patients.

\section{Acknowledgments}

This study was supported by a grant from the Korea Healthcare Technology R\&D Project, Ministry of Health and Welfare, Republic of Korea (HI10C2020). The Ministry of Health and Welfare had no further role in study design, collection, analysis, or interpretation of data, writing of the report, or the decision to submit the paper for publication.

Woo YS, Kim JB, Lee MS, Kim JM, Yim HW, and Jun TY have no conflicts of interest. McIntyre RS has received research grant support from Lundbeck, Astra Zeneca, Pfizer, Shire, Otsuka, Bristol Myers Squibb, National Institute of Mental Health, Stanley Medical Research Institute, Canadian Institutes for Health Research, and The Brain and Behavior Research Foundation. Dr. McIntyre has also received speaker/consultant fees from Lundbeck, Pfizer, Astra Zeneca, Elli Lilly, Janssen Ortho, Sunovion, Takeda, Forest, Otsuka, Bristol Myers Squibb and Shire.

\section{REFERENCES}

1. Alonso J, Angermeyer MC, Bernert S, Bruffaerts R, Brugha $\mathrm{TS}$, Bryson $\mathrm{H}$, et al. Prevalence of mental disorders in Europe: results from the European Study of the Epidemiology of Mental Disorders (ESEMeD) project. Acta Psychiatr Scand Suppl 2004;(420):21-27.

2. Alegria M, Takeuchi D, Canino G, Duan N, Shrout P, Meng $\mathrm{XL}$, et al. Considering context, place and culture: the National Latino and Asian American Study. Int J Methods Psychiatr Res 2004;13:208-220.

3. Takeuchi DT, Chung RC, Lin KM, Shen H, Kurasaki K, Chun CA, et al. Lifetime and twelve-month prevalence rates of major depressive episodes and dysthymia among Chinese Americans in Los Angeles. Am J Psychiatry 1998;155:14071414.

4. Ferrari AJ, Somerville AJ, Baxter AJ, Norman R, Patten SB, Vos $\mathrm{T}$, et al. Global variation in the prevalence and incidence of major depressive disorder: a systematic review of the epidemiological literature. Psychol Med 2013;43:471481. 
5. Weissman MM, Bland RC, Canino GJ, Faravelli C, Greenwald S, Hwu HG, et al. Cross-national epidemiology of major depression and bipolar disorder. JAMA 1996;276: 293-299.

6. Yeung A, Chang D. Mood disorders in Asians. Asian $J$ Psychiatr 2014;7:71-73.

7. Iwata N, Buka S. Racelethnicity and depressive symptoms: a cross-cultural/ethnic comparison among university students in East Asia, North and South America. Soc Sci Med 2002; 55:2243-2252.

8. Cho MJ, Kim KH. Use of the Center for Epidemiologic Studies Depression (CES-D) scale in Korea. J Nerv Ment Dis 1998;186:304-310.

9. Lin KM. Biological differences in depression and anxiety across races and ethnic groups. J Clin Psychiatry 2001;62 Suppl 13:13-9; discussion 20-21.

10. Darmansjah I, Muchtar A. Dose-response variation among different populations. Clin Pharmacol Ther 1992;52:449452.

11. Woo YS, Shim IH, Lee SY, Lee DB, Kim MD, Jung YE, et al. Dose trends of aripiprazole from 2004 to 2014 in psychiatric inpatients in Korea. Clin Psychopharmacol Neurosci 2017;15:177-180.

12. Mundo E, Walker M, Cate T, Macciardi F, Kennedy JL. The role of serotonin transporter protein gene in antidepressantinduced mania in bipolar disorder: preliminary findings. Arch Gen Psychiatry 2001;58:539-544.

13. Zanardi R, Serretti A, Rossini D, Franchini L, Cusin C, Lattuada E, et al. Factors affecting fluvoxamine antidepressant activity: influence of pindolol and 5-HTTLPR in delusional and nondelusional depression. Biol Psychiatry 2001;50:323330.

14. Perlis RH, Mischoulon D, Smoller JW, Wan YJ, LamonFava S, Lin KM, et al. Serotonin transporter polymorphisms and adverse effects with fluoxetine treatment. Biol Psychiatry 2003:54:879-883

15. Kim K, Johnson JA, Derendorf H. Differences in drug pharmacokinetics between East Asians and Caucasians and the role of genetic polymorphisms. J Clin Pharmacol 2004; 44:1083-1105.

16. Palmatier MA, Kang AM, Kidd KK. Global variation in the frequencies of functionally different catechol-O-methyltransferase alleles. Biol Psychiatry 1999;46:557-567.

17. Zhang M. Major depressive disorder treatment guidelines in China. J Clin Psychiatry 2010;71 Suppl E1:e06.

18. Higuchi T. Major depressive disorder treatment guidelines in Japan. J Clin Psychiatry 2010;71 Suppl E1:e05.

19. Seok Seo J, Rim Song H, Bin Lee H, Park YM, Hong JW, $\mathrm{Kim} \mathrm{W}$, et al. The Korean medication algorithm for depressive disorder: second revision. J Affect Disord 2014; 167:312-321

20. Won E, Park SC, Han KM, Sung SH, Lee HY, Paik JW, et al. Evidence-based, pharmacological treatment guideline for depression in Korea, revised edition. J Korean Med Sci 2014;29:468-484.

21. Treuer T, Liu CY, Salazar G, Kongsakon R, Jia F, Habil $\mathrm{H}$, et al. Use of antidepressants in the treatment of depression in Asia: guidelines, clinical evidence, and experience revisited. Asia Pac Psychiatry 2013;5:219-230.

22. Suzuki A, Aoshima T, Fukasawa T, Yoshida K, Higuchi $\mathrm{H}$, Shimizu T, et al. A three-factor model of the MADRS in major depressive disorder. Depress Anxiety 2005;21:95-97.

23. Sheehan DV. Sheehan disability scale. In: Rush AJ, Pincus $H A$, First MB, Blacker D, Endicott J, Keith SJ, et al., editors. Handbook of psychiatric measures. Washington, DC:American
Psychiatric Association;2000. p.113-115.

24. Lingjaerde O, Ahlfors UG, Bech P, Dencker SJ, Elgen K. The UKU side effect rating scale. A new comprehensive rating scale for psychotropic drugs and a cross-sectional study of side effects in neuroleptic-treated patients. Acta Psychiatr Scand Suppl 1987;334:1-100.

25. Hayasaka Y, Purgato M, Magni LR, Ogawa Y, Takeshima N, Cipriani A, et al. Dose equivalents of antidepressants: Evidence-based recommendations from randomized controlled trials. J Affect Disord 2015;180:179-184.

26. Cipriani A, Furukawa TA, Salanti G, Geddes JR, Higgins $\mathrm{JP}$, Churchill R, et al. Comparative efficacy and acceptability of 12 new-generation antidepressants: a multipletreatments meta-analysis. Lancet 2009;373:746-758.

27. Purgato M, Papola D, Gastaldon C, Trespidi C, Magni LR, Rizzo C, et al. Paroxetine versus other anti-depressive agents for depression. Cochrane Database Syst Rev 2014; (4): $C D 006531$.

28. Hong Ng C, Norman TR, Naing KO, Schweitzer I, Kong Wai Ho B, Fan A, et al. A comparative study of sertraline dosages, plasma concentrations, efficacy and adverse reactions in Chinese versus Caucasian patients. Int Clin Psychopharmacol 2006;21:87-92.

29. Chi MH, Lee SY, Chang HH, Yang YK, Lin E, Chen PS. Comparison of antidepressant efficacy-related SNPs among Taiwanese and four populations in the HapMap database. J Formos Med Assoc 2011;110:478-482.

30. Preissner SC, Hoffmann MF, Preissner R, Dunkel M, Gewiess A, Preissner S. Polymorphic cytochrome P450 enzymes (CYPS) and their role in personalized therapy. PLoS One 2013;8:e82562.

31. Sanchez C, Reines EH, Montgomery SA. A comparative review of escitalopram, paroxetine, and sertraline: Are they all alike? Int Clin Psychopharmacol 2014;29:185-196.

32. Rao N. The clinical pharmacokinetics of escitalopram. Clin Pharmacokinet 2007;46:281-290.

33. Jornil J, Jensen KG, Larsen F, Linnet K. Identification of cytochrome P450 isoforms involved in the metabolism of paroxetine and estimation of their importance for human paroxetine metabolism using a population-based simulator. Drug Metab Dispos 2010;38:376-385.

34. Magalhães P, Alves G, Llerena A, Falcão A. Venlafaxine pharmacokinetics focused on drug metabolism and potential biomarkers. Drug Metabol Drug Interact 2014;29:129-141.

35. Spina E, de Leon J. Clinical applications of CYP genotyping in psychiatry. J Neural Transm (Vienna) 2015;122:5-28.

36. Chen PY, Wang SC, Poland RE, Lin KM. Biological variations in depression and anxiety between East and West. CNS Neurosci Ther 2009;15:283-294.

37. Kirchheiner J, Nickchen K, Bauer M, Wong ML, Licinio J, Roots I, et al. Pharmacogenetics of antidepressants and antipsychotics: the contribution of allelic variations to the phenotype of drug response. Mol Psychiatry 2004;9:442473.

38. Lee MS. Role of genetic polymorphisms related to neurotransmitters and cytochrome P-450 enzymes in response to antidepressants. Drugs Today (Barc) 2007;43:569-581.

39. Lobello KW, Preskorn SH, Guico-Pabia CJ, Jiang Q, Paul $\mathrm{J}$, Nichols AI, et al. Cytochrome P450 2D6 phenotype predicts antidepressant efficacy of venlafaxine: a secondary analysis of 4 studies in major depressive disorder. J Clin Psychiatry 2010;71:1482-1487.

40. Mulder H, Wilmink FW, Beumer TL, Tamminga WJ, Jedema JN, Egberts AC. The association between cytochrome P450 $2 D 6$ genotype and prescription patterns of 
antipsychotic and antidepressant drugs in hospitalized psychiatric patients: a retrospective follow-up study. J Clin Psychopharmacol 2005;25:188-191.

41. D'Empaire I, Guico-Pabia CJ, Preskorn SH. Antidepressant treatment and altered CYP2D6 activity: are pharmacokinetic variations clinically relevant? J Psychiatr Pract 2011;17:330-339

42. Kalow W, Otton SV, Kadar D, Endrenyi L, Inaba T. Ethnic difference in drug metabolism: debrisoquine 4-hydroxylation in Caucasians and Orientals. Can J Physiol Pharmacol 1980;58:1142-1144.

43. Bousman CA, Sarris J, Won ES, Chang HS, Singh A, Lee $\mathrm{HY}$, et al. Escitalopram efficacy in depression: a crossethnicity examination of the serotonin transporter promoter polymorphism. J Clin Psychopharmacol 2014;34:645-648.

44. Kang RH, Wong ML, Choi MJ, Paik JW, Lee MS. Association study of the serotonin transporter promoter polymorphism and mirtazapine antidepressant response in major depressive disorder. Prog Neuropsychopharmacol Biol Psychiatry 2007;31:1317-1321.

45. Kim DK, Lim SW, Lee S, Sohn SE, Kim S, Hahn CG, et al. Serotonin transporter gene polymorphism and antidepressant response. Neuroreport 2000;11:215-219.

46. Yoshida K, Ito K, Sato K, Takahashi H, Kamata M, Higuchi $\mathrm{H}$, et al. Influence of the serotonin transporter gene-linked polymorphic region on the antidepressant response to fluvoxamine in Japanese depressed patients. Prog Neuropsychopharmacol Biol Psychiatry 2002;26:383-386.

47. Yoshimura R, Umene-Nakano W, Suzuki A, Ueda N, Miyamoto K, Ikenouchi-Sugita A, et al. Rapid response to paroxetine is associated with plasma paroxetine levels at 4 but not 8 weeks of treatment, and is independent of serotonin transporter promoter polymorphism in Japanese depressed patients. Hum Psychopharmacol 2009;24:489494.

48. Chen H, Guarnaccia PJ, Chung H. Self-attention as a mediator of cultural influences on depression. Int $J$ Soc Psychiatry 2003;49:192-203.

49. Waza K, Graham AV, Zyzanski SJ, Inoue K. Comparison of symptoms in Japanese and American depressed primary care patients. Fam Pract 1999;16:528-533.

50. Parker G, Cheah YC, Roy K. Do the Chinese somatize depression? A cross-cultural study. Soc Psychiatry Psychiatr Epidemiol 2001;36:287-293.

51. Georg Hsu LK, Wan YM, Chang H, Summergrad P, Tsang BY, Chen H. Stigma of depression is more severe in Chinese Americans than Caucasian Americans. Psychiatry 2008; 71 : 210-218.
52. Trivedi MH, Rush AJ, Wisniewski SR, Nierenberg AA, Warden D, Ritz L, et al. Evaluation of outcomes with citalopram for depression using measurement-based care in STAR*D: implications for clinical practice. Am J Psychiatry 2006; 163:28-40.

53. Iosifescu DV, Nierenberg AA, Alpert JE, Smith M, Bitran $\mathrm{S}$, Dording $\mathrm{C}$, et al. The impact of medical comorbidity on acute treatment in major depressive disorder. Am J Psychiatry 2003;160:2122-2127.

54. Papakostas GI, Petersen T, Denninger J, Sonawalla SB, Mahal Y, Alpert JE, et al. Somatic symptoms in treatmentresistant depression. Psychiatry Res 2003;118:39-45.

55. Klengel T, Heck A, Pfister H, Brückl T, Hennings JM, Menke A, et al. Somatization in major depression--clinical features and genetic associations. Acta Psychiatr Scand 2011;124:317-328.

56. Serretti A, Mandelli L, Lorenzi C, Pirovano A, Olgiati P, Colombo $\mathrm{C}$, et al. Serotonin transporter gene influences the time course of improvement of "core" depressive and somatic anxiety symptoms during treatment with SSRIs for recurrent mood disorders. Psychiatry Res 2007;149:185193.

57. Taylor SE, Way BM, Welch WT, Hilmert CJ, Lehman BJ, Eisenberger NI. Early family environment, current adversity, the serotonin transporter promoter polymorphism, and depressive symptomatology. Biol Psychiatry 2006;60:671-676.

58. Nock MK, Deming CA, Fullerton CS, Gilman SE, Goldenberg M, Kessler RC, et al. Suicide among soldiers: a review of psychosocial risk and protective factors. Psychiatry 2013;76:97-125.

59. Hansen RA, Moore CG, Dusetzina SB, Leinwand BI, Gartlehner G, Gaynes BN. Controlling for drug dose in systematic review and meta-analysis: a case study of the effect of antidepressant dose. Med Decis Making 2009;29: 91-103.

60. Warden D, Rush AJ, Wisniewski SR, Lesser IM, Thase ME, Balasubramani GK, et al. Income and attrition in the treatment of depression: a STAR*D report. Depress Anxiety 2009;26:622-633.

61. Warden D, Rush AJ, Carmody TJ, Kashner TM, Biggs MM, Crismon ML, et al. Predictors of attrition during one year of depression treatment: a roadmap to personalized intervention. J Psychiatr Pract 2009;15:113-124.

62. Turvey CL, Jogerst G, Kim MY, Frolova E. Cultural differences in depression-related stigma in late-life: a comparison between the USA, Russia, and South Korea. Int Psychogeriatr 2012;24:1642-1647. 